Article

\title{
On the Continuous Cancellative Semigroups on a Real Interval and on a Circle and Some Symmetry Issues
}

\author{
Mariusz Bajger ${ }^{1,+}+\mathbb{D}$, Janusz Brzdęk ${ }^{2, *,+} \mathbb{D}$, El-sayed El-hady ${ }^{3,4,+}+\mathbb{D}$ and Eliza Jabłońska ${ }^{5,+}$ \\ 1 College of Science and Engineering, Flinders University, Adelaide, SA 5042, Australia; \\ mariusz.bajger@flinders.edu.au \\ 2 Faculty of Applied Mathematics, AGH University of Science and Technology, Mickiewicza 30, \\ 30-059 Kraków, Poland \\ 3 Mathematics Department, College of Science, Jouf University, Sakaka 72388, Saudi Arabia; \\ eaelhady@ju.edu.sa or elsayed_elhady@ci.suez.edu.eg \\ 4 Basic Science Department, Faculty of Computers and Informatics, Suez Canal University, \\ Ismailia 41522, Egypt \\ 5 Institute of Mathematics, Pedagogical University of Cracow, Podchorążych 2, 30-084 Kraków, Poland; \\ eliza.jablonska@up.krakow.pl \\ * Correspondence: brzdek@agh.edu.pl \\ + These authors contributed equally to this work.
}

Received: 29 October 2020; Accepted: 27 November 2020; Published: 29 November 2020

\begin{abstract}
Let $S$ denote the unit circle on the complex plane and $\star: S^{2} \rightarrow S$ be a continuous binary, associative and cancellative operation. From some already known results, it can be deduced that the semigroup $(S, \star)$ is isomorphic to the group $(S, \cdot)$; thus, it is a group, where $\cdot$ is the usual multiplication of complex numbers. However, an elementary construction of such isomorphism has not been published so far. We present an elementary construction of all such continuous isomorphisms $F$ from $(S, \cdot)$ into $(S, \star)$ and obtain, in this way, the following description of operation $\star: x \star y=$ $F\left(F^{-1}(x) \cdot F^{-1}(y)\right)$ for $x, y \in S$. We also provide some applications of that result and underline some symmetry issues, which arise between the consequences of it and of the analogous outcome for the real interval and which concern functional equations. In particular, we show how to use the result in the descriptions of the continuous flows and minimal homeomorphisms on $S$.
\end{abstract}

Keywords: continuous binary operation; semigroup; cancellative operation; unit circle; real interval; homomorphism; minimal homeomorphism; continuous flow

MSC: 22A15; 30D05; 39B12; 39B22; 39B32

\section{Introduction}

Let $(X, 0)$ and $(X, *)$ be groupoids (i.e., $X$ is a nonempty set and $\circ, *: X^{2} \rightarrow X$ are binary operations in $X)$. If there exists a bijection $h: X \rightarrow X$ such that

$$
x \circ y=h^{-1}(h(x) * h(y)) \text { for } x, y \in X,
$$

then we say that the pair $(h, *)$ induces $\circ$. It is easily seen that (1) is equivalent to the following property

$$
h(x \circ y)=h(x) * h(y) \quad \text { for } x, y \in X,
$$

which means that $h$ is an isomorphism of $(X, 0)$ into $(X, *)$. 
Recall yet that a groupoid $(X, 0)$ is cancellative if operation $\circ$ is cancellative, i.e.,

$$
x \circ y \neq x \circ z \quad \text { and } \quad y \circ x \neq z \circ x,
$$

for all $x, y, z \in X$ with $y \neq z$. A groupoid $(X, 0)$ is a semigroup if operation $\circ$ is associative, i.e., $x \circ(y \circ z)=(x \circ y) \circ z$ for every $x, y, z \in X$.

Let $S$ denote the unit circle of the complex plane $\mathbb{C}$ and $\cdot$ be the usual multiplication of complex numbers. The main result of this paper is an elementary construction of homeomorphisms $h: S \rightarrow S$ such that the pair $(h, \cdot)$ induces a given binary, continuous, associative, and cancellative operation on $S$, where the topology in $S$ is induced by the usual topology of the plane. As far as we know it is the only such proof.

This paper is partly of expository type because we also show several interesting applications of the main outcome and underline some symmetry issues concerning functional equations, which arise between those applications and some consequences of the following well known result of J. Aczél $[1,2]$ (analogous result was obtained in Reference [3]; also see Reference [4] for a simpler proof).

Theorem 1. Let I be a real nontrivial interval and $\circ: I \times I \rightarrow I$ be a binary operation that is continuous, associative, and cancellative. Then, there exist an infinite real interval $J$ and a homeomorphism $H: I \rightarrow J$ with

$$
x \circ y=H^{-1}(H(x)+H(y)) \text { for } x, y \in I .
$$

Note that property (4) means, in particular, that $H(x)+H(y) \in J$ for every $x, y \in I$, i.e., $z+w \in J$ for every $z, w \in J$. Therefore, $J$ must be either the set of reals $\mathbb{R}$ or of one of the following forms: $(a, \infty)$, $[a, \infty),(-\infty,-a),(-\infty,-a]$ with some real $a \geq 0$.

Next, it is clear that every operation of form (4) must be cancellative. So, without the cancellativity assumption, the result is not true, and, for instance, the natural operation

$$
x \circ y:=\max \{x, y\} \text { for } x, y \in I \text {, }
$$

which is associative and continuous, but not cancellative, cannot be represented in form (4). The same is true for $x \circ y:=\min \{x, y\}$ for $x, y \in I$.

In the terms of functional equations, Theorem 1 is about continuous solutions $A: I^{2} \rightarrow I$ (if we write $A(x, y):=x \circ y$ ) of the following associativity equation:

$$
A(x, A(y, z))=A(A(x, y), z) \text { for } x, y, z \in I \text {. }
$$

In this case, the cancellativity of $\circ$ is equivalent to the injectivity of $A$ with respect to either variable, which means the strict monotonicity with respect to either variable (because of the assumed continuity of $A$ ). Similar problem without the assumption of strict monotonicity (i.e., the cancellativity of the corresponding binary operation), was considered in Reference [5] (also see Reference [6]), and, under some additional assumptions, the following representation was obtained

$$
A(x, y)=g(H(x)+H(y)) \text { for } x, y \in I,
$$

with some continuous injection $H: I \rightarrow \mathbb{R}$, where $g:[0, \infty] \rightarrow I$ is a pseudoinverse of $H$ (see Reference [5] for more details). Clearly, representation (4) is actually (7) with $g=H^{-1}$. For further, more general investigations of that subject, we refer to References [7-9].

Let us add that solutions $A:[0,1]^{2} \rightarrow[0,1]$ to the associativity Equation (6) are important in statistical metric spaces and are called triangular norms or shortly $t$-norms (cf., e.g., References $[6,10,11])$; we also refer to Reference [12] for the notion of copulas.

A result analogous to Theorem 1, for the unit circle, has the subsequent form. 
Theorem 2. Let a binary operation $\star: S \times S \rightarrow S$ be continuous, associative, and cancellative. Then, there exist exactly two homeomorphisms $F_{0}, G_{0}: S \rightarrow S$ such that

$$
x \star y=F_{0}\left(F_{0}^{-1}(x) \cdot F_{0}^{-1}(y)\right)=G_{0}\left(G_{0}^{-1}(x) \cdot G_{0}^{-1}(y)\right) \text { for } x, y \in S .
$$

In particular, $F_{0}(x)=G_{0}(\bar{x})$ for $x \in S$, where $\bar{x}$ is the complex conjugate of $x$.

Theorem 2 can be derived (with some additional reasoning) from Reference [13] (Theorems 1.10 and 1.13) (continuous, compact and cancellative semigroup is a topological group) and Reference [14] (Theorem 2) (connected topological group, which contains a neighbourhood of the neutral element that is homeomorphic to an open real interval, is isomorphic either to the additive group of reals $(\mathbb{R},+)$ or to the factor group $(\mathbb{R} / \mathbb{Z},+)$, where $\mathbb{Z}$ denotes the set of integers).

However, as we will see in the section with applications, it is useful to know how to construct the function $F_{0}$ (and thus also $G_{0}$ ). In the case of Theorem 1 , the form of $H$ can be deduced from the proofs in References [2,4]. We show in Section 5 that a symmetric reasoning, with a reasonably elementary and simple construction of the functions $F_{0}$ and $G_{0}$, works for Theorem 2.

Finally, let us mention that the form of $A$ in (7) reminds about a well-known problem of representing functions with several variables by functions in one variable, whether under some regularity assumptions or not (see References [5,15-17] for more details).

For further information on topological semigroups (also on historical background) we refer to References $[13,18,19]$.

The paper is divided into 6 sections. The next section contains some observations concerning symmetry issue arising between Theorems 1 and 2. In Section 3, we present several applications of both theorems. Section 4, titled Auxiliary Results, contains a series of definitions, lemmas, remarks, and corollaries, leading to the final reasoning contained in Section 5, titled The Proof of Theorems 2, and being the proper proof of the theorem with a description of function $F_{0}$. The sixth section (the last one) presents some concluding remarks.

\section{Some Remarks on Symmetry Issues}

Let us observe that the statements of Theorems 1 and 2 imply that both operations, $\circ$ and $\star$, must be commutative (symmetric). Moreover, in the next section we demonstrate that some applications of those theorems yield several somewhat symmetric results concerning functional equations. But, first, let us note some symmetry deficiencies between Theorems 1 and 2.

Remark 1. S is compact, while the interval J in Theorem 1 must be infinite and therefore not compact, which means that I cannot be compact. So, every continuous semigroup on a compact interval is not cancellative.

Remark 2. In Theorem 2 there exist exactly two functions, $F_{0}$ and $G_{0}$, satisfying (8). The situation in the case of Theorem 1 (for a real interval) is somewhat different. Namely, let $F: I \rightarrow \mathbb{R}$ also be a continuous injection such that $x \circ y=F^{-1}(F(x)+F(y))$ for $x, y \in I$ (which means, in particular, that $F(x)+F(y) \in F(I)$ for $x, y \in I)$. Then

$$
H^{-1}(H(x)+H(y))=x \circ y=F^{-1}(F(x)+F(y)) \text { for } x, y \in I,
$$

whence

$$
F \circ H^{-1}(H(x)+H(y))=F \circ H^{-1}(H(x))+F \circ H^{-1}(H(y)) \text { for } x, y \in I,
$$

which means that the function $h=F \circ H^{-1}: J \rightarrow J$ is a homeomorphism satisfying

$$
h(z+w)=h(z)+h(w) \text { for } z, w \in J .
$$


Consequently, $h(z) \equiv c z$ with some real $c \neq 0$ (see, e.g., Reference [20]), which means (with $z=H(x)$ ) that

$$
F(x)=c H(x) \text { for } x \in I \text {. }
$$

Remark 3. Note that, even if $I=\mathbb{R}$ in Theorem $1,(I, 0)$ does not need to be a group, contrary to the situation on the circle. This happens in the case when $H(\mathbb{R})=(0, \infty)$.

\section{Applications}

Now, we show some simple applications of Theorems 1 and 2 in functional equations. They show some symmetries and some lack of symmetry between those two cases of the continuous cancellative semigroups on a real interval and on the unit circle. As they are clearly visible, we do not discuss them in detail.

Let us begin with the following auxiliary useful result in Reference [21] (p. 155).

Lemma 1. Let $\mathbb{K} \in\{\mathbb{R}, \mathbb{C}\}$. Then, a continuous function $g: \mathbb{K} \rightarrow \mathbb{C}$ satisfies the functional equation

$$
g(t+s)=g(t) \cdot g(s) \text { for } t, s \in \mathbb{K}
$$

if and only if

(I) in the case $\mathbb{K}=\mathbb{R}$, there is $a \in \mathbb{C}$ with $g(s)=e^{a s}$ for $s \in \mathbb{R}$;

(II) in the case $\mathbb{K}=\mathbb{C}$, there are $b, c \in \mathbb{C}$ with $g(s)=e^{c s+b \bar{s}}$ for $s \in \mathbb{C}$.

The next lemma seems to be well known, but, for the convenience of readers, we present a short proof of it.

Lemma 2. Let $A: S \rightarrow S$ be a continuous solution of the equation

$$
A(v \cdot w)=A(v) \cdot A(w) \text { for } v, w \in S .
$$

Then, there is a real constant $d$ such that $A\left(e^{2 \pi i t}\right)=e^{2 \pi i d t}$ for $t \in \mathbb{R}$.

Proof. Define a function $B: \mathbb{R} \rightarrow S$ by $B(t)=A\left(e^{2 \pi i t}\right)$ for $t \in \mathbb{R}$. Then, $B$ is continuous and, by (14), $B(t+s)=A\left(e^{2 \pi i(t+s)}\right)=A\left(e^{2 \pi i t}\right) \cdot A\left(e^{2 \pi i s}\right)=B(t) \cdot B(s)$ for $s, t \in \mathbb{R}$. Consequently, by Lemma 1 , there is $a \in \mathbb{C}$ such that $B(t)=e^{a t}$ for $t \in \mathbb{R}$. Since $B(\mathbb{R}) \subset S$, the real part of $a$ must be equal 0 . So $d:=\frac{a}{2 \pi i} \in \mathbb{R}$ and consequently $B(t)=e^{2 \pi i d t}$ for $t \in \mathbb{R}$. Hence, $A\left(e^{2 \pi i t}\right)=B(t)=e^{2 \pi i d t}$ for $t \in \mathbb{R}$.

Now, we are in a position to present the mentioned before applications. As far as we know, they are new results, never published so far.

Proposition 1. Let a binary operation $\star: S \times S \rightarrow S$ be associative, continuous and cancellative. Let $\mathbb{K} \in$ $\{\mathbb{R}, \mathbb{C}\}$ and $F_{0}: S \rightarrow S$ be a homeomorphism with

$$
x \star y=F_{0}\left(F_{0}^{-1}(x) \cdot F_{0}^{-1}(y)\right) \text { for } x, y \in S .
$$

Then, a continuous function $f: \mathbb{K} \rightarrow S$ fulfills the equation

$$
f(t+s)=f(t) \star f(s) \text { for } s, t \in \mathbb{K},
$$

if and only if there is $d \in \mathbb{K}$ such that,

(a) in the case $\mathbb{K}=\mathbb{R}, f(s)=F_{0}\left(e^{i d s}\right)$ for $s \in \mathbb{R}$; 
(b) in the case $\mathbb{K}=\mathbb{C}, f(s)=F_{0}\left(e^{i(d s+\bar{d} \bar{s})}\right)$ for $s \in \mathbb{C}$.

Proof. Let $f: \mathbb{K} \rightarrow S$ be a continuous function that fulfills Equation (16). Then, by (15),

$$
f(t+s)=f(t) \star f(s)=F_{0}\left(F_{0}^{-1}(f(t)) \cdot F_{0}^{-1}(f(t))\right) \text { for } s, t \in \mathbb{K},
$$

whence the function $h: \mathbb{K} \rightarrow S, h(t)=F_{0}^{-1}(f(t))$ for $t \in \mathbb{K}$, fulfills Equation (13). Hence, by Lemma 1, conditions (I) and (II) are valid. Clearly, $e^{a s} \in S$ for $s \in \mathbb{R}$ if and only if $a i \in \mathbb{R}$. Moreover, it is easy to check that $e^{c s+b \bar{s}} \in S$ for $s \in \mathbb{C}$ if and only if $c=\bar{b}$. This implies statements (a) and (b).

Now, suppose that $f: \mathbb{R} \rightarrow S$ has the form depicted by (a). Then, in view of (15),

$$
f(t+s)=F_{0}\left(e^{i d(t+s)}\right)=F_{0}\left(e^{i d t} \cdot e^{i d s}\right)=F_{0}\left(e^{i d t}\right) \cdot F_{0}\left(e^{i d s}\right)=f(t) \star f(s) \text { for } s, t \in \mathbb{R} .
$$

The case of $(b)$ is analogous.

Proposition 2. Let I be a nontrivial real interval and a binary operation $\circ: I \times I \rightarrow I$ be associative, continuous, and cancellative. Let $\mathbb{K} \in\{\mathbb{R}, \mathbb{C}\}$ and $H: I \rightarrow \mathbb{R}$ be a continuous injection satisfying (4). Then, the functional equation

$$
f(t+s)=f(t) \circ f(s) \text { for } s, t \in \mathbb{K}
$$

has a non-constant continuous solution $f: \mathbb{K} \rightarrow I$ if and only if $H$ is bijective.

Moreover, if $H$ is bijective, then a continuous function $f: \mathbb{K} \rightarrow$ I fulfills Equation (19) if and only if there is $d \in \mathbb{K}$ such that,

(i) in the case $\mathbb{K}=\mathbb{R}, f(s)=H^{-1}(d s)$ for $s \in \mathbb{R}$;

(ii) in the case $\mathbb{K}=\mathbb{C}, f(s)=H^{-1}(d s+\bar{d} \bar{s})$ for $s \in \mathbb{C}$.

Proof. Let $f: \mathbb{K} \rightarrow I$ be a continuous solution of Equation (19). Then, the function $h: \mathbb{K} \rightarrow I$, $h(t)=H(f(t))$ for $t \in \mathbb{K}$, fulfills the equation

$$
h(t+s)=h(t)+h(s) \text { for } s, t \in \mathbb{K} .
$$

Moreover, $h$ is continuous.

In the case $\mathbb{K}=\mathbb{R}$ there is $d \in \mathbb{R}$ such that $h(s)=d s$ for $s \in \mathbb{R}$, which implies that either $d=0$ or $H$ is surjective and $f(s)=H^{-1}(d s)$ for $s \in \mathbb{R}$.

In the case $\mathbb{K}=\mathbb{C}$ there are $d_{1}, d_{2} \in \mathbb{K}$ such that $h(s)=d_{1} \Re s+d_{2} \Im s$ for $s \in \mathbb{C}$, where $\Re s$ and $\Im s$ denote the real and imaginary parts of a complex number $s$. Write $d=\frac{1}{2}\left(d_{1}-i d_{2}\right)$. Then, it is easily seen that $h(s)=d s+\bar{d} \bar{s}$ for $s \in \mathbb{C}$, which implies that either $d=0$ or $H$ is surjective and $f(s)=H^{-1}(d s+\bar{d} \bar{s})$ for $s \in \mathbb{C}$.

Now, we see that, in the case where $H$ is bijective, we obtain statements (i) and (ii). Moreover, it is easy to check functions depicted in statements (i) and (ii) are solutions to (19).

Next, suppose that Equation (19) has a non-constant continuous solution $f: \mathbb{K} \rightarrow I$. Then, as we have already observed, in the case $\mathbb{K}=\mathbb{R}$ there is $d \in \mathbb{R}$ such that $H(f(s))=d s$ for $s \in \mathbb{R}$. Since $f$ is not constant and $H$ is injective, this means that $d \neq 0$ and consequently $H$ is surjective. If $\mathbb{K}=\mathbb{C}$, then we show the surjectivity of $H$ in a similar way.

Finally, note that, if $H$ is bijective, then functions depicted by statements (i) and (ii), with $d \neq 0$, are non-constant continuous solutions to (19).

Proposition 3. Let I be a nontrivial real interval and binary operations $\circ: I \times I \rightarrow I$ and $\star: S \times S \rightarrow S$ be associative, continuous and cancellative. Let $H: I \rightarrow \mathbb{R}$ and $F_{0}: S \rightarrow S$ be such that (4) and (15) hold. Then, the following two statements are valid. 
(A) A continuous function $f: I \rightarrow S$ fulfills the functional equation

$$
f(t \circ s)=f(t) \star f(s) \text { for } s, t \in I,
$$

if and only if there is $d \in \mathbb{R}$ such that $f(t)=F_{0}\left(e^{i d H(t)}\right)$ for $t \in \mathbb{R}$.

(B) Every continuous function $f: S \rightarrow$ I fulfilling the functional equation

$$
f(u \star v)=f(u) \circ f(v) \text { for } u, v \in S,
$$

is constant.

Proof. First we prove (A). So, let $f: I \rightarrow S$ be a continuous function that fulfills functional Equation (21). Then, in view of (4) and (15),

$$
f\left(H^{-1}(H(t)+H(s))\right)=F_{0}\left(F_{0}^{-1}(f(t)) \cdot F_{0}^{-1}(f(s))\right) \text { for } s, t \in I,
$$

which means that the function $h: H(I) \rightarrow S, h(t)=F_{0}^{-1}\left(f\left(H^{-1}(t)\right)\right)$ for $t \in H(I)$ is continuous and satisfies the equation

$$
h(s+t)=h(s) \cdot h(t) \text { for } s, t \in H(I) .
$$

Let $h_{0}: \mathbb{R} \rightarrow S$ be the solution of

$$
h_{0}(s+t)=h_{0}(s) \cdot h_{0}(t) \text { for } s, t \in \mathbb{R}
$$

such that $h(t)=h_{0}(t)$ for $t \in H(I)$ (we define $h_{0}$ by: $h_{0}(s-t):=h(s) \cdot h(t)^{-1}$ for $s, t \in H(I)$ ). According to Proposition 1 there is $d \in \mathbb{R}$ such that $h_{0}(t)=e^{i d t}$ for $t \in \mathbb{R}$, whence $h(t)=e^{i d t}$ for $t \in H(I)$. Consequently,

$$
f(t)=F_{0}(h(H(t)))=F_{0}\left(e^{i d H(t)}\right) \text { for } t \in I .
$$

The converse is easy to check.

Now, we prove (B). Let $f: S \rightarrow I$ be a continuous solution to (22). Then, in view of (4) and (15),

$$
f\left(F_{0}\left(F_{0}^{-1}(u) \cdot F_{0}^{-1}(v)\right)\right)=H^{-1}(H(f(u))+H(f(v))) \text { for } u, v \in S,
$$

whence the function $h: S \rightarrow \mathbb{R}, h(u)=H\left(f\left(F_{0}(u)\right)\right)$ for $u \in F_{0}^{-1}(S)=S$, is continuous and satisfies the equation

$$
h(u \cdot v)=h(u)+h(v) \text { for } u, v \in S .
$$

Thus, the set $h(S)$ is compact (because $S$ is compact and $h$ is continuous) and it is a subgroup of $(\mathbb{R},+)$. The only such subgroup is the trivial one $\{0\}$, which means that $h(S)=\{0\}$. Since $F_{0}$ is bijective and $H$ is injective, $f$ must be constant.

Using Theorem 2 and some results from Reference [22] we also get the following proposition on the minimal homeomorphisms on $S$. Let us recall (see Reference [22] (Ch.1 §1)) that, if $f: S \rightarrow S$, then a set $A \subseteq S$ is called $f$-invariant provided $f(A) \subseteq A$. A homeomorphism $f: S \rightarrow S$ is minimal if $S$ does not contain any non-empty, proper, closed $f$-invariant subset (see References $[23,24]$ for some related results).

Proposition 4. A function $T: S \rightarrow S$ is a minimal homeomorphism if and only if there exist $a \in S$ and $a$ continuous, associative, and cancellative binary operation $\star: S \times S \rightarrow S$ such that

$$
\begin{aligned}
& T(x)=x \star a \text { for } x \in S, \\
& a \neq a^{n} \text { for } n \in \mathbb{N}, n>1,
\end{aligned}
$$


where $a^{1}=a$ and $a^{n+1}=a^{n} \star a$ for $n \in \mathbb{N}(\mathbb{N}$ denotes the set of positive integers).

Proof. Assume that $T: S \rightarrow S$ is a minimal homeomorphism. Then, there exist an irrational real number $c$ and a homeomorphism $\psi: S \rightarrow S$ with $\psi(T(x))=e^{2 \pi i c} \cdot \psi(x)$ for $x \in S$ (see Reference [22] (Ch.3 §3 Th.1,3)). Define a binary operation $\star: S \times S \rightarrow S$ by

$$
u \star v=\psi^{-1}(\psi(u) \cdot \psi(v)) \text { for } u, v \in S,
$$

and put $a:=\psi^{-1}\left(e^{2 \pi i c}\right)$. Then, $T(x)=\psi^{-1}\left(\psi(x) \cdot e^{2 \pi i c}\right)=x \star a$ for $x \in S$. Moreover, since $\psi^{-1}$ is injective and $c$ is irrational, we have

$$
a^{n}=\psi^{-1}\left(e^{2 \pi i n c}\right) \neq \psi^{-1}\left(e^{2 \pi i c}\right)=a \text { for } n \in \mathbb{N}, n>1
$$

which means that (30) holds.

Now, suppose that $T: S \rightarrow S$ is of form (29), where $\star: S \times S \rightarrow S$ is a continuous, associative, and cancellative binary operation. In view of Theorem 2, there exists a homeomorphism $F_{0}: S \rightarrow S$ such that (15) is valid. Consequently, $T$ is a homeomorphism and $F_{0}^{-1}(T(x))=F_{0}^{-1}(x \star a)=F_{0}^{-1}(x)$. $F_{0}^{-1}(a)$ for $x \in S$. Let $c \in[0,1)$ be such that $F_{0}^{-1}(a)=e^{2 \pi i c}$. Then,

$$
T^{n}(x)=x \star a^{n}=F_{0}\left(F_{0}^{-1}(x) \cdot F_{0}^{-1}\left(a^{n}\right)\right)=F_{0}\left(F_{0}^{-1}(x) \cdot e^{2 \pi i n c}\right) \text { for } x \in S, n \in \mathbb{Z} .
$$

Now, we show that $c$ is irrational. So, for the proof by contradiction suppose that there are $n, k \in \mathbb{N}$ with $(n-1) c=k$. Then, $a^{n}=F_{0}\left(e^{2 \pi i n c}\right)=F_{0}\left(e^{2 \pi i k} \cdot e^{2 \pi i c}\right)=F_{0}\left(e^{2 \pi i c}\right)=a$. This is a contradiction to (30).

Thus, we have proved that $c$ is irrational. Hence, it follows that the set $\left\{e^{2 \pi i n c}: n \in \mathbb{Z}\right\}$ is dense in $S$; thus, by (33), the set $\left\{T^{n}(x): n \in \mathbb{Z}\right\}$ is dense in $S$ for every $x \in S$, which means that $T$ is a minimal homeomorphism. This ends the proof.

Before our last proposition in this section, let us remind the notion of continuous flow on a topological space $X$. Namely (cf. References $[13,25]$ ), if $f_{t}: X \rightarrow X$ for $t \in \mathbb{R}$ is a family of maps such that $f_{t+s}=f_{t} \circ f_{s}$ for all $t, s \in \mathbb{R}, f_{0}$ is the identity map on $X$ and the mapping $\Phi: X \times \mathbb{R} \rightarrow X$, given by $\Phi(x, t)=f_{t}(x)$, is continuous, then we say that the family $f_{t}: X \rightarrow X$ for $t \in \mathbb{R}$ is a continuous flow on $X$.

Observe that, if $f_{t}: X \rightarrow X$ for $t \in \mathbb{R}$ is a continuous flow on a topological space $X$, then $\Phi$ : $X \times \mathbb{R} \rightarrow X, \Phi(x, t)=f_{t}(x)$, is a continuous solution of the translation functional equation

$$
\Phi(\Phi(x, t), s)=\Phi(x, t+s) \text { for } x \in X, t \in \mathbb{R} .
$$

The last proposition in this section shows that the continuous, associative, and cancellative binary operations on $S$ can be used in a description of the continuous flows on $S$ (cf. References [26,27]) and therefore also in a description of the continuous solutions $\Phi: S \times \mathbb{R} \rightarrow S$ to the translation functional equation.

Proposition 5. A family of continuous functions $\left\{T^{t}: S \rightarrow S: t \in \mathbb{R}\right\}$ is a continuous flow such that either $T^{1}=i d$ (the identity function on $S$ ) or $T^{1}(x) \neq x$ for $x \in S$ if and only if there exist a continuous, associative, and cancellative operation $\star: S \times S \rightarrow S$ and a continuous solution $f: \mathbb{R} \rightarrow S$ of the functional equation

$$
f(t+s)=f(t) \star f(s) \text { for } s, t \in \mathbb{R}
$$

such that

$$
T^{t}(x)=x \star f(t) \text { for } x \in S, t \in \mathbb{R}
$$


Proof. Let $\left\{T^{t}: S \rightarrow S: t \in \mathbb{R}\right\}$ be a continuous flow such that either $T^{1}=i d$ or $T^{1}(x) \neq x$ for $x \in S$. Then, by [13] (Theorem 2), there exist $d \in \mathbb{R}$ and an orientation preserving homeomorphism $\psi: S \rightarrow S$ with $T^{t}(x)=\psi^{-1}\left(\psi(x) \cdot e^{i d t}\right)$ for $x \in S, t \in \mathbb{R}$.

Let $\star: S \times S \rightarrow S$ be the operation given by (31). Then, $\star$ is continuous and $(S, \star)$ is a group. Next, the function $f: \mathbb{R} \rightarrow S, f(t)=\psi^{-1}\left(e^{i d t}\right)$ for $t \in \mathbb{R}$, fulfills Equation (35) (because $\psi(f(t))=e^{i d t}$ for $t \in \mathbb{R}$ ) and

$$
T^{t}(x)=\psi^{-1}\left(\psi(x) \cdot e^{i d t}\right)=\psi^{-1}(\psi(x) \cdot \psi(f(t)))=x \star f(t) \text { for } x \in S, t \in \mathbb{R} .
$$

Conversely, suppose that (36) holds. Then, it is easily seen that $\left\{T^{t}: t \in \mathbb{R}\right\}$ is a continuous flow. Further, by Theorem $2,(S, \star)$ is a group. So, if there is $x_{0} \in S$ such that $T^{1}\left(x_{0}\right)=x_{0}$, then, by (36), $x_{0} \star f(1)=x_{0}$, whence $f(1)$ is the neutral element of $(S, \star)$. Thus, (36) implies that $T^{1}=\mathrm{id}$. This completes the proof.

Finally, let us mention that somewhat related issues in particle physics can be found in Reference [28]. Moreover, it seems that Theorem 2 can be applied in finding continuous solutions $f: S \rightarrow S$ of some suitable functional equations analogously as Theorem 1 has been used in References [29-31] for real functions.

\section{Auxiliary Results}

In this section, we provide information and several observations necessary in the proof of Theorem 2 for the construction of function $F_{0}$. They are presented in a series of lemmas, remarks, and corollaries. We start with some notations and definitions.

We define an order in $S$ as follows:

$$
\begin{aligned}
& u \prec v \Leftrightarrow \operatorname{Arg} u<\operatorname{Arg} v, \\
& u \preceq v \Leftrightarrow \operatorname{Arg} u \leq \operatorname{Arg} v
\end{aligned}
$$

for $u, v \in S$, where $\operatorname{Arg} u \in[0,2 \pi)$ stands for the argument of the complex number $u$. For every $u, v \in S, u \prec v$, we write

$$
\begin{gathered}
a(u, v):=\{s \in S: u \prec s \prec v\}, \\
c(u, v):=\{s \in S: s \prec u \text { or } v \prec s\} .
\end{gathered}
$$

It is known (cf., e.g., Reference [22] (Chap. 2)) that, for every homeomorphism $T: S \rightarrow S$, there exists a unique homeomorphism $f: \mathbb{R} \rightarrow \mathbb{R}$ satisfying $T\left(e^{2 \pi i x}\right)=e^{2 \pi i f(x)}$ for $x \in \mathbb{R}$. If $f$ is increasing (decreasing), then we say that homeomorphism $T$ preserves (reverses) orientation. Note that every homeomorphism $T: S \rightarrow S$ preserves or reverses orientation.

Now, we will prove several auxiliary lemmas, corollaries and remarks.

So, let $(S, \star)$ be as in Theorem 2. Then, $(S, \star)$ is a topological group (see Reference [13] (Theorems 1.10 and 1.13)). Denote by $e$ the neutral element of $(S, \star)$ and define $\circ: S \times S \rightarrow S$ by

$$
u \circ v=\frac{1}{e} \cdot((e \cdot u) \star(e \cdot v)) \text { for } u, v \in S .
$$

Remark 4. It is easily seen that the mapping $\gamma: S \rightarrow S, \gamma(u)=e \cdot u$ for $u \in S$, is an isomorphism from $(S, o)$ onto $(S, \star)$. So, $(S, 0)$ is a group with the neutral element equal 1 . Next, $\gamma$ is a homeomorphism and

$$
u \circ v=\gamma^{-1}(\gamma(u) \star \gamma(v)) \text { for } u, v \in S,
$$

whence $(S, \circ)$ is a topological group. For every $u \in S$, by $u^{-1}$, we always denote the inverse element to $u$ in the group $(S, \circ)$ and $J: S \rightarrow S$ is defined by $J(v)=v^{-1}$ for $v \in S$. 
In what follows, $u^{1}:=u$ and $u^{n+1}:=u \circ u^{n}$ for $u \in S$ and $n \in \mathbb{N}$. Clearly, by the associativity of the operation, we have

$$
u^{n+m}=u^{n} \circ u^{m} \text { for } u \in S, n, m \in \mathbb{N} .
$$

Next, given $u \in S$, we define functions $L_{u}, R_{u}: S \rightarrow S$ by the formulas:

$$
L_{u}(s)=u \circ s, R_{u}(s)=s \circ u \text { for } s \in S .
$$

Remark 5. Let $u \in S \backslash\{1\}$. Since $(S, \circ)$ is a topological group, $L_{u}$ and $R_{u}$ are homeomorphisms without fixed points and therefore preserve orientation (see Reference [13] (Remark 3)).

Lemma 3. There exists $u_{0} \in S$ such that $u_{0} \prec u_{0}^{-1}$.

Proof. First, suppose that $u=u^{-1}$ for every $u \in S$, that is $u^{2}=1$ for every $u \in S$. Let $u, v \in S$ and $1 \prec$ $u \prec v$. Then, by and Remark 5, we have $v \circ a(1, v)=c(1, v)$, where $v \circ a(1, v):=\{v \circ s: s \in a(1, v)\}$. This means that $v \prec v \circ u$.

On the other hand, $c(1, u) \circ u=a(1, u)$, and, consequently, $v \circ u \prec u$, a contradiction because $u \prec v$. So, there exists $v_{0} \in S$ such that $v_{0}^{2} \neq 1$. Putting $u_{0}=v_{0}^{-1}$, we have either $v_{0} \prec v_{0}^{-1}$ or $u_{0} \prec u_{0}^{-1}$.

Lemma 4. If $u, v \in S$ and $1 \prec u \prec v$, then $v^{-1} \prec u^{-1}$.

Proof. It follows from Lemma 3 that there exists $u_{0} \in S$ such that $u_{0} \prec u_{0}^{-1}$. Since $J\left(u_{0}^{-1}\right)=u_{0} \prec$ $u_{0}^{-1}=J\left(u_{0}\right)$ and $J(1)=1, J$ reverses orientation. Thus, the assertion follows from the fact that $1 \notin J(a(u, v))$.

Lemma 5. Let $s, u, v \in S, u \prec v$, and $1 \prec s \prec v^{-1}$. Then, $u \circ s \prec v \circ s$ and $s \circ u \prec s \circ v$.

Proof. Lemma 4 implies that $v \prec s^{-1}$. Thus, $1 \notin R_{s}(a(u, v))$ and $1 \notin L_{s}(a(u, v))$. The lemma results now from Remark 5.

From Lemma 5 (for $u=1$ ), we obtain the following

Corollary 1. If $s, v \in S \backslash\{1\}$ and $s \prec v^{-1}$, then $s \prec v \circ s$ and $s \prec s \circ v$.

Lemma 6. Let $v \in S, v \prec v^{-1}$. Then:

(a) $v \prec v^{2}$;

(b) if $t, s \in S, t \prec s \prec v$, then $t^{2} \prec s^{2}$.

Proof. Assertion (a) results from Corollary 1 (with $v=s$ ). For the proof of (b), fix $e \prec t \prec s \prec v$. Then, by Lemma 4 , we get $t \prec s \prec v \prec v^{-1} \prec s^{-1} \prec t^{-1}$. Hence, Lemma 6 gives

$$
t^{2}=t \circ t \prec t \circ s \prec s \circ s=s^{2} .
$$

This ends the proof.

Lemma 7. Assume that $u \in S$ and $u \prec u^{-1}$. Then, for every $x \in S, x \prec u^{2}$, there exists exactly one $y \in S$, $y \prec u$, such that $y^{2}=x$. Moreover, $y \prec x$.

Proof. Define a function $q: a(1, u) \rightarrow S, q(s)=s^{2}$. Then, $q$ is continuous and, on account of Lemma $6 \mathrm{~b}$, $q$ is injective. Thus, $q(a(1, u))=a\left(1, u^{2}\right)$ and $q^{-1}(x) \in a(1, u)$ for every $x \in a\left(1, u^{2}\right)$. Fix $x \in a\left(1, u^{2}\right)$. By Lemma $4, y:=q^{-1}(x) \prec u \prec u^{-1} \prec y^{-1}$. Consequently, in view of Lemma $6 \mathrm{a}, y \prec x$, which ends the proof. 
Lemma 8. Let $u \in S, u \prec u^{-1}$. Then, for every $n \in \mathbb{N}$, the set $A_{n}(u):=\left\{y \in S: y^{2^{n}}=u\right\}$ is non-empty and has the smallest element $p_{n}(u) \prec u$. Moreover, $p_{n+1}(u) \prec p_{n}(u)$ and $\left(p_{n+1}(u)\right)^{2}=p_{n}(u)$ for $n \in \mathbb{N}$.

Proof. The proof is by induction with respect to $n$.

The case $n=1$ results from Lemma 7. Fix $n \in \mathbb{N}$ and assume that $A_{n}(u) \neq \varnothing$ and there exists the smallest element $p_{n}(u)$ of $A_{n}(u)$ with $p_{n}(u) \prec u$. Then, according to Lemma 7 , there is exactly one element $y \prec p_{n}(u)$ such that $y^{2}=p_{n}(u)$. Note that $y^{2^{n+1}}=\left(y^{2}\right)^{2^{n}}=\left[p_{n}(u)\right]^{2^{n}}=u$. Thus, $y \in A_{n+1}(u)$.

Suppose that there is $z \in A_{n+1}(u)$ with $z \prec y$. Then, $u=z^{2^{n+1}}=\left(z^{2}\right)^{2^{n}}$, whence $z^{2} \in A_{n}(u)$. Moreover, by Lemma $6 \mathrm{~b}, z^{2} \prec y^{2}=p_{n}(u)$, a contradiction. Consequently, $y$ is the smallest element of $A_{n+1}(u), y^{2}=p_{n}(u)$, and $y \prec p_{n}(u)$. This completes the proof.

Remark 6. Fix $u_{0} \in S$ with $u_{0} \prec u_{0}^{-1}$ (see Lemma 3). From Lemma 8, by induction, we get $\left(p_{n+m}\left(u_{0}\right)\right)^{2^{m}}=$ $p_{n}\left(u_{0}\right)$ for $n, m \in \mathbb{N}_{0}:=\mathbb{N} \cup\{0\}$, where $p_{0}\left(u_{0}\right)=u_{0}$. Hence, for $j, k \in \mathbb{N}_{0}, k \leq j$, we have

$$
p_{k}\left(u_{0}\right) \circ p_{j}\left(u_{0}\right)=\left[p_{j}\left(u_{0}\right)\right]^{2^{j-k}} \circ p_{j}\left(u_{0}\right)=\left[p_{j}\left(u_{0}\right)\right]^{2^{j-k}+1}=p_{j}\left(u_{0}\right) \circ p_{k}\left(u_{0}\right) .
$$

Let

$$
D:=\left\{\sum_{j=0}^{k} 2^{-j} x_{j}: x_{0} \in \mathbb{N}_{0}, k \in \mathbb{N}, x_{j} \in\{0,1\} \text { for } j=1,2, \ldots, k\right\} .
$$

Note that, for every $x \in D_{0}:=D \backslash \mathbb{N}_{0}$, there are unique $k(x) \in \mathbb{N}, m(x) \in \mathbb{N}_{0}$ and $x_{1}, \ldots, x_{k(x)} \in\{0,1\}$ such that $x_{k(x)}=1$ and $x=m(x)+\sum_{j=1}^{k(x)} 2^{-j} x_{j}$. Thus, we can define a function $d: D \rightarrow S$ by

$$
d(m)=u_{0}^{m}, \quad d\left(m+\sum_{j=1}^{k} 2^{-j} x_{j}\right)=u_{0}^{m} \circ \prod_{j=1}^{k} r_{j}\left(x_{j}\right)
$$

for $k, m \in \mathbb{N}_{0}, k>0, x_{1}, \ldots, x_{k} \in\{0,1\}, x_{k}=1$, where $u_{0}:=1, r_{j}(1)=p_{j}\left(u_{0}\right), r_{j}(0)=1$ for $j \in \mathbb{N}$ and $\prod_{j=1}^{k} r_{j}\left(x_{j}\right):=r_{1}\left(x_{1}\right) \circ \ldots \circ r_{k}\left(x_{k}\right)$.

In what follows, we assume that $u_{0} \in S$ is fixed and $u_{0} \prec u_{0}^{-1}$. Moreover, the function $d$ is defined as above. Now, we prove some properties of $d$. We start with the following

Lemma 9. Let $D$ and $d: D \rightarrow S$ be defined as in Remark 6. Then, $d(x+y)=d(x) \circ d(y)$ for every $x, y \in D$.

Proof. In view of Remark 6, for every $x \in D_{0}$, there exist unique $k(x) \in \mathbb{N}, m(x) \in \mathbb{N}_{0}$, and $x_{1}, \ldots, x_{k(x)} \in\{0,1\}$ with $x_{k(x)}=1$ and $x=m(x)+\sum_{i=1}^{k(x)} 2^{-i} x_{i}$. If $x \in \mathbb{N}_{0}$, then we write $k(x):=0$ and $m(x):=x$.

The proof is by induction with respect to $p(x, y):=\max \{k(x), k(y)\}$. The case $p(x, y)=0$ is trivial because, then,

$$
d(x+y)=d(m(x)+m(y))=u_{0}^{m(x)+m(y)}=u_{0}^{m(x)} \circ u_{0}^{m(y)}=d(x) \circ d(y) .
$$

So, fix $n \in \mathbb{N}_{0}$ and assume that the assertion holds for every $x, y \in D$ with $p(x, y) \leq n$. Take $x, y \in D$ with $p(x, y)=n+1$. Since, by (47), $d(x) \circ d(y)=d(y) \circ d(x)$, it suffices to consider the following two cases.

Case I: $k(x)=k(y)=n+1$. Then,

$$
x=m(x)+\sum_{i=1}^{n+1} 2^{-i} x_{i}, \quad y=m(y)+\sum_{i=1}^{n+1} 2^{-i} y_{i}
$$


with $x_{n+1}=y_{n+1}=1$. Write

$$
x_{0}=m(x)+\sum_{i=1}^{n} 2^{-i} x_{i}, \quad y_{0}=m(y)+\sum_{i=1}^{n} 2^{-i} y_{i}
$$

Clearly $\max \left\{k\left(x_{0}\right), k\left(y_{0}\right), k\left(x_{0}+y_{0}\right)\right\} \leq n$. So, in view of Remark 6, Lemma 8 , and the induction hypothesis, we have

$$
\begin{aligned}
d(x+y) & =d\left(m(x)+\sum_{i=1}^{n+1} 2^{-i} x_{i}+m(y)+\sum_{i=1}^{n+1} 2^{-i} y_{i}\right) \\
& =d\left(m(x)+\sum_{i=1}^{n} 2^{-i} x_{i}+m(y)+\sum_{i=1}^{n} 2^{-i} y_{i}+2^{-n}\right) \\
& =d\left(x_{0}+y_{0}\right) \circ d\left(2^{-n}\right)=d\left(x_{0}\right) \circ d\left(y_{0}\right) \circ p_{n}\left(u_{0}\right) \\
& =u_{0}^{m(x)} \circ \prod_{i=1}^{k\left(x_{0}\right)} r_{i}\left(x_{i}\right) \circ u_{0}^{m(y)} \circ \prod_{i=1}^{k\left(y_{0}\right)} r_{i}\left(y_{i}\right) \circ p_{n+1}\left(u_{0}\right) \circ p_{n+1}\left(u_{0}\right) \\
& =u_{0}^{m(x)} \circ \prod_{i=1}^{k\left(x_{0}\right)} r_{i}\left(x_{i}\right) \circ p_{n+1}\left(u_{0}\right) \circ u_{0}^{m(y)} \circ \prod_{i=1}^{k\left(y_{0}\right)} r_{i}\left(y_{i}\right) \circ p_{n+1}\left(u_{0}\right) \\
& =d(x) \circ d(y) .
\end{aligned}
$$

Case II: $k(x)<k(y)=n+1$. Let $z=\sum_{i=1}^{k(x)} 2^{-i} x_{i}+\sum_{i=1}^{n} 2^{-i} y_{i}$ and $y_{0}=\sum_{i=1}^{n} 2^{-i} y_{i}$. Then, there exist $k(z), m(z) \in \mathbb{N} \cup\{0\}, k(z) \leq n$, and $z_{i} \in\{0,1\}$ for $i=1, \ldots, k(z), z_{k(z)}=1$ such that $z=m(z)+\sum_{i=1}^{k(z)} 2^{-i} z_{i}$. Hence, by Remark 6 and the induction hypothesis, we obtain

$$
\begin{aligned}
& d(x+y)=d\left(m(x)+\sum_{i=1}^{k(x)} 2^{-i} x_{i}+m(y)+\sum_{i=1}^{n+1} 2^{-i} y_{i}\right) \\
& =d\left(m(x)+m(y)+z+2^{-n-1}\right) \\
& =d\left(m(x)+m(y)+m(z)+\sum_{i=1}^{k(z)} 2^{-i} z_{i}+2^{-n-1}\right) \\
& =u_{0}^{m(x)+m(y)+m(z)} \circ \prod_{i=1}^{k(z)} r_{i}\left(z_{i}\right) \circ d\left(2^{-n-1}\right) \\
& =d\left(m(x)+m(y)+m(z)+\sum_{i=1}^{k(z)} 2^{-i} z_{i}\right) \circ p_{n+1}\left(u_{0}\right) \\
& =d\left(m(x)+\sum_{i=1}^{k(x)} 2^{-i} x_{i}+m(y)+\sum_{i=1}^{k\left(y_{0}\right)} 2^{-i} y_{i}\right) \circ p_{n+1}\left(u_{0}\right) \\
& =d\left(m(x)+\sum_{i=1}^{k(x)} 2^{-i} x_{i}\right) \circ d\left(m(y)+\sum_{i=1}^{k\left(y_{0}\right)} 2^{-i} y_{i}\right) \circ p_{n+1}\left(u_{0}\right) \\
& =d(x) \circ u^{m(y)} \circ \prod_{i=1}^{k\left(y_{0}\right)} r_{i}\left(y_{i}\right) \circ p_{n+1}\left(u_{0}\right)=d(x) \circ d(y) \text {. }
\end{aligned}
$$

Lemma 10. Let $D$ and $d: D \rightarrow S$ be the same way as in Lemma 9. Then, $d(x) \prec u_{0}$ for every $x \in D, x<1$.

Proof. Clearly, $d(0)=u_{0}^{0}=1 \prec u_{0}$. Next, for every $x \in D_{0}, x<1$, there are unique $k(x) \in \mathbb{N}_{0}$, $x_{1}, \ldots, x_{k(x)} \in\{0,1\}, x_{k(x)}=1$, with $x=\sum_{i=1}^{k(x)} 2^{-i} x_{i}$.

The proof is by induction with respect to $k(x)$. The case $k(x)=1$ results from Lemma 8 . Fix $n \in \mathbb{N}$ and assume that the assertion holds for every $x \in D_{0}, x<1$, with $k(x) \leq n$. Let $y \in D_{0}, k(y)=n+1$, and $z=2^{-n-1}$. From the induction hypothesis and Lemma 4 , we obtain

$$
\prod_{i=1}^{n} r_{i}\left(y_{i}\right) \prec u_{0} \prec u_{0}^{-1} \prec p_{n}\left(u_{0}\right)^{-1}
$$


because Lemma 8 yields $p_{n}\left(u_{0}\right) \prec u_{0}$. By Lemma 8, we also get $p_{n+1}\left(u_{0}\right) \prec p_{n}\left(u_{0}\right) \prec u_{0} \prec u_{0}^{-1} \prec$ $p_{n}\left(u_{0}\right)^{-1}$. Hence, in view of the induction hypothesis, Lemma 5 and Lemma 9 , we have

$$
\begin{aligned}
d(y) & =\prod_{i=1}^{n+1} r_{i}\left(y_{i}\right)=\prod_{i=1}^{n} r_{i}\left(y_{i}\right) \circ p_{n+1}\left(u_{0}\right) \\
& \prec \prod_{i=1}^{n} r_{i}\left(y_{i}\right) \circ p_{n}\left(u_{0}\right)=d\left(\sum_{i=1}^{n} 2^{-i} y_{i}+2^{-n}\right) \preceq u_{0} .
\end{aligned}
$$

This completes the proof.

Lemma 11. Let $D$ and $d: D \rightarrow S$ be the same way as in Lemma 9, $x, y \in D$ and $x<y<1$. Then, $d(x) \prec d(y)$.

Proof. By Lemmas 10 and 4 , we have $d(x) \prec u_{0} \prec d(y-x)^{-1}$. Thus, on account of Corollary 1 and Lemma $9, d(x) \prec d(x) \circ d(y-x)=d(y)$. This ends the proof.

Let

$$
M:=\sup \{z \in D: \text { if } x, y \in D \text { and } x<y<z \text {, then } d(x) \prec d(y)\} \text {. }
$$

Lemma 12. If $M$ is defined by (57) with $D$ and $d$ the same way as in Lemma 9 , then $M \in[1, \infty)$.

Proof. From Lemma 11, we get immediately $M \geq 1$. We prove that $M$ is a finite number.

Suppose that $u_{0}^{n} \prec u_{0}^{-1}$ for every $n \in \mathbb{N}$. Then, on account of Corollary $1, u_{0}^{n} \prec u_{0}^{n+1}$ for $n \in \mathbb{N}$ and consequently there exists $s=\lim _{n \rightarrow \infty} u_{0}^{n} \preceq u_{0}^{-1}$.

If $s=u_{0}^{-1}$, then, in view of the continuity of the operation, we have

$$
u_{0}^{-1}=s=\lim _{n \rightarrow \infty} u_{0}^{n}=u_{0} \circ \lim _{n \rightarrow \infty} u_{0}^{n}=u_{0} \circ s=1,
$$

a contradiction.

If $s \prec u_{0}^{-1}$, then, by Corollary $1, s \prec u_{0} \circ s=u_{0} \circ \lim _{n \rightarrow \infty} u_{0}^{n}=\lim _{n \rightarrow \infty} u_{0}^{n}=s$, a contradiction, too.

In this way we have proved that there is $k \in \mathbb{N}$ with $u_{0}^{-1} \preceq u_{0}^{k}$. Thus, writing $n:=1+\max \{m \in$ $\left.\mathbb{N}: u_{0}^{m} \prec u_{0}^{-1}\right\}$, we get $u_{0}^{j} \prec u_{0}^{-1}$ for $j=1, \ldots, n-1$ and $u_{0}^{-1} \preceq u_{0}^{n}$. Observe that the set $A=a\left(u_{0}^{n-1}, u_{0}^{n}\right) \cup\left\{u_{0}^{n}\right\}$ is connected, $A \neq S$, and $u_{0}^{-1} \in A$. Hence, $1 \in L_{u_{0}}(A)$ and consequently, by Remark $5, u_{0}^{n+1} \prec u_{0}^{n}$, which means that $M \leq n+1$. This ends the proof.

Lemma 13. Let $D$ and $d: D \rightarrow S$ be the same way as in Lemma 9 and $M$ be given by (57). Then, the following two statements are valid.

(a) For every $s \in S \backslash\{1\}$, there is $x \in D, x<1$, with $d(x) \prec s$.

(b) The set $U:=\{d(x): x \in D, x<M\}$ is dense in $S$.

Proof. (a) Put $r:=\inf \left\{p_{n}\left(u_{0}\right): n \in \mathbb{N}\right\}$ (with respect to the order $\prec$ ) and suppose that $1 \prec r$. From Lemma 8 we get $r=\lim _{n \rightarrow \infty} p_{n}\left(u_{0}\right)$ and, by Lemma 7 with $u=r, p_{1}(r) \prec r \prec u_{0} \prec u_{0}^{-1} \prec r^{-1}$. Thus, there exists $t \in S$ with $p_{1}(r) \prec t \prec r$. Hence, on account of Lemma $6 \mathrm{~b}$ with $v=r, r \prec t^{2}$. By the definition of $r$ and Lemma 8, there exists $m \in \mathbb{N}$ such that $r \prec p_{m}\left(u_{0}\right) \prec t^{2}$. Next, in view of Lemma 7 , with $u=t$, there exists $q \in a(1, t)$ with $q^{2}=p_{m}\left(u_{0}\right)$, whence, by Lemma $8, p_{m+1}\left(u_{0}\right) \preceq q \prec t \prec r$, which contradicts the definition of $r$.

(b) For the proof by contradiction, suppose that there exist $v_{1}, v_{2} \in S$ such that $1 \prec v_{1} \prec v_{2}$ and $U \cap a\left(v_{1}, v_{2}\right)=\varnothing$. First, consider the case where $t \prec v_{1}$ for every $t \in U$. Put $s:=\sup U$ (with respect to the order $\prec$ ) and $s_{0}:=\min \left\{s, s^{-1}\right\}$. By a) and Lemma 11 , there exists $m \in \mathbb{N}$ such that $d\left(2^{-m}\right) \prec s_{0}$. 
Let $D^{\prime}:=\left[0, M+2^{-m}\right) \cap D$ and fix $x, y \in D^{\prime}$ such that $x<y, 2^{-m}<y$. Put $x_{0}=\max \left\{2^{-m}, x\right\}$. Then, by Lemma 12, there exist $x^{\prime}, y^{\prime} \in[0, M) \cap D$ and $z, w \in D$ such that $w \leq z<2^{-m} \leq x^{\prime}<y^{\prime}$ and $x_{0}=x^{\prime}+w, y=y^{\prime}+z$, which, in virtue of the definition of $M$ and Lemma 4, means that $d\left(x^{\prime}\right) \prec d\left(y^{\prime}\right)$,

$$
d(w) \preceq d(z) \prec d\left(2^{-m}\right) \prec s_{0} \preceq s^{-1} \prec d\left(y^{\prime}\right)^{-1} \prec d\left(x^{\prime}\right)^{-1},
$$

and $d\left(x^{\prime}\right) \prec d(z)^{-1}$. Thus, on account of Lemmas 5 and 9, we obtain

$$
d\left(x_{0}\right)=d\left(x^{\prime}\right) \circ d(w) \preceq d\left(x^{\prime}\right) \circ d(z) \prec d\left(y^{\prime}\right) \circ d(z)=d\left(y^{\prime}+z\right)=d(y) .
$$

Since, according to the definition of $M, d(x) \prec d(y)$ for $x, y \in D, x<y \leq 2^{-m}$, in this way, we have proved that $d(x) \prec d(y)$ for every $x, y \in D^{\prime}$ with $x<y$. This contradicts the definition of $M$.

Now, consider the case where there is $t \in U$ such that $v_{2} \prec t$. Put $s_{1}=\sup \left\{t \in U: t \prec v_{1}\right\}$, $s_{2}=\inf \left\{t \in U: v_{2} \prec t\right\}$ (with respect to $\prec$ ), and $L:=\sup \left\{x \in D \cap[0, M): d(x) \prec v_{1}\right\}$. Let $V$ be a neighbourhood (in $S$ ) of $s_{1}$ such that $x \prec v_{2}$ for every $x \in V$. Choose a neighbourhood $V_{0}$ of 1 such that $V_{0} \circ s_{1} \subset V$. It results from a) and Lemma 11 that there exists $q \in \mathbb{N}$ with $d\left(2^{-q}\right) \prec s_{1}^{-1}$, $d\left(2^{-q}\right) \in V_{0}$, and $2^{-q}<M-L$. Thus, $d\left(2^{-q}\right) \circ s_{1} \in V$. Moreover, Lemma 4 and Corollary 1 give $s_{1} \prec d\left(2^{-q}\right) \circ s_{1} \prec s_{2}$. Let $V_{1}$ be a neighbourhood of $d\left(2^{-q}\right) \circ s_{1}$ such that $s_{1} \prec x \prec s_{2}$ for every $x \in V_{1}$. By the definition of $s_{1}$, there is $t_{0} \in U$ with $t_{0} \preceq s_{1}$ and $d\left(2^{-q}\right) \circ t_{0} \in V_{1}$, which means that $s_{1} \prec d\left(2^{-q}\right) \circ t_{0} \prec s_{2}$. Clearly, $t_{0}=d(r)$ for some $r \in(0, L] \cap D$. Since $2^{-q}+r<M$ and, by Lemma 9 , $d\left(2^{-q}+r\right)=d\left(2^{-q}\right) \circ t_{0} \in U$, we obtain a contradiction with the definition of $s_{1}$ and $s_{2}$.

Define a function $\bar{d}:[0, M] \rightarrow S$ by the formula

$$
\bar{d}(x)= \begin{cases}\sup \{d(y): y<x, y \in D\} & \text { for } x \in(0, M) \\ 1 & \text { for } x \in\{0, M\}\end{cases}
$$

where $D$ and $d: D \rightarrow S$ are the same way as in Lemma 9 and $M$ is given by (57).

As an immediate consequence of the definition and Lemma 13, we have the following.

Corollary 2. Let $\bar{d}:[0, M] \rightarrow S$ be defined as above, where $M$ is given by (57). Then, $\left.\bar{d}\right|_{[0, M)}$ is a strictly increasing function (with respect to the order $\prec$ in $S$ ).

Lemma 14. Let $M$ and $\bar{d}:[0, M] \rightarrow S$ be as in Corollary 2. Then, $\bar{d}$ is a continuous function.

Proof. Using Lemma 13a and Corollary 2, it is easily seen that $\bar{d}$ is continuous at 0 . Next, we show that $\bar{d}$ is continuous at $M$. So, fix an increasing sequence $\left\{r_{n}\right\}_{n \in \mathbb{N}}$ in $D$ such that $\lim _{n \rightarrow \infty} r_{n}=M$. Then, according to the definitions of $M$ and $\bar{d}$ and Lemma $13 \mathrm{~b}$, for every $t \in S$, there is $n \in \mathbb{N}$ with $t \prec \bar{d}\left(r_{n}\right)$, which means that $\lim _{n \rightarrow \infty} \bar{d}\left(r_{n}\right)=1$. Hence, by Corollary $2, \bar{d}$ is continuous at $M$.

To complete the proof suppose that $\bar{d}$ is discontinuous at a point $x_{0} \in(0, M)$. Then, by Corollary 2 and Lemma 13b, there exist $z_{1}, z_{2} \in(0, M)$ with $z_{1}<z_{2}$ and $x_{0} \in\left[z_{1}, z_{2}\right]$, and a sequence $\left\{r_{n}\right\}_{n \in \mathbb{N}}$ in $D \cap\left((0, M) \backslash\left\{x_{0}\right\}\right)$ such that $\lim _{n \rightarrow \infty} r_{n}=x_{0}$ and

$$
r_{n}<x_{0}<r_{n}+2^{-n}, \quad \bar{d}\left(r_{n}\right) \prec \bar{d}\left(z_{1}\right) \prec \bar{d}\left(z_{2}\right) \prec \bar{d}\left(r_{n}+2^{-n}\right), \quad n \in \mathbb{N} .
$$

Hence, $r_{n}<z_{1}<z_{2}<r_{n}+2^{-n}$ for $n \in \mathbb{N}$. Since

$$
x_{0}=\lim _{n \rightarrow \infty} r_{n} \leq z_{1}<z_{2} \leq \lim _{n \rightarrow \infty}\left(r_{n}+2^{-n}\right)=x_{0},
$$

we obtain a contradiction. This ends the proof.

Lemma 15. Let $M$ and $\bar{d}:[0, M] \rightarrow S$ be as in Corollary 2 and $x, y \in[0, M]$. Then: 
(a) if $x+y \leq M$, then $\bar{d}(x+y)=\bar{d}(x) \circ \bar{d}(y)$;

(b) if $M<x+y$, then $\bar{d}(x+y-M)=\bar{d}(x) \circ \bar{d}(y)$.

Proof. (a) Let $\left\{x_{n}\right\}_{n \in \mathbb{N}},\left\{y_{n}\right\}_{n \in \mathbb{N}}$ be sequences in $D \backslash\{x, y\}$ such that $x_{n}+y_{n}<M$ for $n \in \mathbb{N}$ and $\lim _{n \rightarrow \infty} x_{n}=x, \lim _{n \rightarrow \infty} y_{n}=y$. By the continuity of $\circ$ and Lemmas 9 and 14 , we have

$$
\begin{aligned}
\bar{d}(x) \circ \bar{d}(y) & =\lim _{n \rightarrow \infty} \bar{d}\left(x_{n}\right) \circ \lim _{n \rightarrow \infty} \bar{d}\left(y_{n}\right)=\lim _{n \rightarrow \infty} d\left(x_{n}\right) \circ \lim _{n \rightarrow \infty} d\left(y_{n}\right) \\
& =\lim _{n \rightarrow \infty} d\left(x_{n}\right) \circ d\left(y_{n}\right)=\lim _{n \rightarrow \infty} d\left(x_{n}+y_{n}\right) \\
& =\lim _{n \rightarrow \infty} \bar{d}\left(x_{n}+y_{n}\right)=\bar{d}(x+y) .
\end{aligned}
$$

(b) Let $\left\{x_{n}\right\}_{n \in \mathbb{N}},\left\{y_{n}\right\}_{n \in \mathbb{N}}$ be increasing sequences in $D \backslash\{x, y\}$ with $\lim _{n \rightarrow \infty} x_{n}=x$, $\lim _{n \rightarrow \infty} y_{n}=y$. Take a sequence $\left\{m_{n}\right\}_{n \in \mathbb{N}}$ in $D \cap(0, M)$ with $\lim _{n \rightarrow \infty} m_{n}=M$ and write $z_{n}:=$ $x_{n}+y_{n}-m_{n}$ for $n \in \mathbb{N}$. Then, $z_{n} \in D$ for $n \in \mathbb{N}$ and, by Lemmas 9 and 14 , we may write

$$
\begin{aligned}
\bar{d}(x) \circ \bar{d}(y) & =\lim _{n \rightarrow \infty} d\left(x_{n}\right) \circ d\left(y_{n}\right)=\lim _{n \rightarrow \infty} d\left(x_{n}+y_{n}\right) \\
& =\lim _{n \rightarrow \infty} d\left(m_{n}+z_{n}\right)=\lim _{n \rightarrow \infty} d\left(m_{n}\right) \circ d\left(z_{n}\right) \\
& =\lim _{n \rightarrow \infty} d\left(m_{n}\right) \circ \lim _{n \rightarrow \infty} d\left(z_{n}\right)=\bar{d}(x+y-M) .
\end{aligned}
$$

This ends the proof.

\section{Proof of Theorem 2}

Now, we are in a position to present the final part of the proof of Theorem 2. In what follows, $M$ and $\bar{d}:[0, M] \rightarrow S$ have the same meaning as in Corollary 2.

Define a function $F: S \rightarrow S$ as follows:

$$
F(v):=\bar{d}\left(\frac{M \operatorname{Arg} v}{2 \pi}\right) \text { for } v \in S .
$$

Then, by Corollary 2, $F$ is strictly increasing (with respect to the order $\prec$ in $S$ ) and, on account of Lemma 14 , it is easily seen that $F$ is continuous. Moreover, it results from Lemma 15 that, for every $v, w \in S$,

$$
\begin{aligned}
F(v \cdot w) & =\bar{d}\left(\frac{M \operatorname{Arg}(v \cdot w)}{2 \pi}\right) \\
& = \begin{cases}\bar{d}\left(\frac{M(\operatorname{Arg} v+\operatorname{Arg} w)}{2 \pi}\right), & \text { if } \operatorname{Arg} v+\operatorname{Arg} w<2 \pi ; \\
\bar{d}\left(\frac{M(\operatorname{Arg} v+\operatorname{Arg} w-2 \pi)}{2 \pi}\right), & \text { if } \operatorname{Arg} v+\operatorname{Arg} w \in[2 \pi, 4 \pi)\end{cases} \\
& =\bar{d}\left(\frac{M \operatorname{Arg} v}{2 \pi}\right) \circ \bar{d}\left(\frac{M \operatorname{Arg} w}{2 \pi}\right)=F(v) \circ F(w) .
\end{aligned}
$$

Consequently, $F$ is a homeomorphism fulfilling

$$
v \circ w=F\left(F^{-1}(v) \cdot F^{-1}(w)\right) \text { for } v, w \in S .
$$


Write $F_{0}:=\gamma \circ F$, where $\gamma: S \rightarrow S$ is defined as in Remark 4. Then, $F_{0}: S \rightarrow S$ is a homeomorphism and, by (68),

$$
\begin{aligned}
x \star y & =\gamma\left(\gamma^{-1}(x) \circ \gamma^{-1}(y)\right)=\gamma\left(F\left(F^{-1}\left(\gamma^{-1}(x)\right) \cdot F^{-1}\left(\gamma^{-1}(y)\right)\right)\right) \\
& =F_{0}\left(F_{0}^{-1}(x) \cdot F_{0}^{-1}(y)\right), \quad x, y \in S .
\end{aligned}
$$

To complete the proof of Theorem 2 suppose that $G_{0}: S \rightarrow S$ is also a homeomorphism such that $x \star y=G_{0}\left(G_{0}^{-1}(x) \cdot G_{0}^{-1}(y)\right)$ for $x, y \in S$. Then, for every $v, w \in S$,

$$
F_{0}(v \cdot w)=F_{0}(v) \star F_{0}(w)=G_{0}\left(G_{0}^{-1}\left(F_{0}(v)\right) \cdot G_{0}^{-1}\left(F_{0}(w)\right)\right),
$$

whence $G_{0}^{-1}\left(F_{0}(v \cdot w)\right)=G_{0}^{-1}\left(F_{0}(v)\right) \cdot G_{0}^{-1}\left(F_{0}(w)\right)$. Putting $A(u)=G_{0}^{-1}\left(F_{0}(u)\right)$ for $u \in S$ we get $F_{0}=G_{0} \circ A$ and

$$
A(v \cdot w)=A(v) \cdot A(w) \text { for } v, w \in S .
$$

Moreover, $A$ is a homeomorphism. Hence, in view of Lemma $2, A(u) \equiv u$ or $A(u) \equiv \bar{u}$. Since it is easily seen that (69) yields

$$
x \star y=G_{0}\left(G_{0}^{-1}(x) \cdot G_{0}^{-1}(y)\right) \text { for } x, y \in S,
$$

this completes the proof.

\section{Conclusions}

Given a binary, continuous, associative, and cancellative operation $\star: S^{2} \rightarrow S$, we presented an elementary construction of all continuous isomorphisms from the group $(S, \cdot)$ onto the semigroup $(S, \star)$, where $S$ is the unit circle on the complex plane and $\cdot$ is the usual multiplication of complex numbers. There are exactly two such isomorphisms $F, G: S \rightarrow S$, and they uniquely determine the form of operation $\star$ in the following way:

$$
x \star y=F\left(F^{-1}(x) F^{-1}(y)\right)=G\left(G^{-1}(x) G^{-1}(y)\right) \text { for } x, y \in S .
$$

Moreover, $F(x)=G(\bar{x})$ for all $x \in S$.

Using this result, we have easily determined all continuous solutions $f: \mathbb{K} \rightarrow S$ of the functional equation

$$
f(x+y)=f(x) \star f(y) \text { for } x, y \in \mathbb{K},
$$

where $\mathbb{K}$ is either the set of reals $\mathbb{R}$ or the set of complex numbers $\mathbb{C}$.

We also provided some further applications of that result in functional equations and showed how to use it in the descriptions of the continuous flows and minimal homeomorphisms on $S$. In particular, we underlined some symmetry issues, which arise between the consequences of the result and of the analogous outcome for the real interval.

It would be interesting to investigate in the future to what extent the statements of Theorems 1 and 2 remain valid if the cancellativity is replaced by the one side cancellativity; for instance, by the left-cancellativity (a groupoid $(X, 0)$ is left-cancellative if $x \circ y \neq x \circ z$ for all $x, y, z \in X$ with $y \neq z$ ).

The next step might be to investigate how much the associativity assumption can be weakened; for instance, to what extent the assumption can be replaced by the square symmetry defined by the formula: $(x \circ x) \circ(y \circ y)=(x \circ y) \circ(x \circ y)$. A natural example of square symmetric operation in $\mathbb{R}$, which is not associative, is given by: $x \star y=a x+b y+c$ for $x, y, z \in \mathbb{R}$, where $a, b, c \in \mathbb{R}$ are fixed and

$$
a c \neq b c, \quad \text { or } \quad a^{2} \neq a, \quad \text { or } \quad b^{2} \neq b .
$$


Such new results would have interesting applications in functional equations in a similar way as Theorem 1 in References [29-31].

Author Contributions: Conceptualization, M.B. and J.B.; methodology, M.B., J.B., E.-s.E.-h., and E.J.; software, M.B., J.B., E.-s.E.-h., and E.J.; validation, M.B., J.B., E.-s.E.-h., and E.J.; formal analysis, M.B., J.B, E.-s.E.-h., and E.J.; investigation, M.B., J.B., E.-s.E.-h., and E.J.; resources, M.B., J.B., E.-s.E.-h., and E.J.; data curation, M.B., J.B., E.-s.E.-h., and E.J.; writing-original draft preparation, M.B. and J.B.; writing-review and editing, M.B., J.B., E.-s.E.-h., and E.J.; visualization, M.B., J.B., E.-s.E.-h., and E.J.; supervision, J.B.; project administration, E.-s.E.-h., and E.J.; funding acquisition, M.B., J.B., E.-s.E.-h., and E.J. All authors have read and agreed to the published version of the manuscript.

Funding: This research received no external funding.

Conflicts of Interest: The authors declare no conflict of interest.

\section{References}

1. Aczél, J. Sur les operations definies pour nombres réels. Bull. Soc. Math. Fr. 1948, 76, 59-64. [CrossRef]

2. Aczél, J. A Short Course on Functional Equations; Reidel: Dordrecht, The Netherlands, 1987.

3. Tamari, D. Caractérisation des semigroupes à un paramètre. Comptes Rendus Acad. Sci. Paris 1949, 228, $1092-1094$.

4. Craigen, R.; Páles, Z. The associativity equation revisited. Aequ. Math. 1989, 37, 306-312. [CrossRef]

5. Cho-Hsin, L. Representation of associative functions. Publ. Math. Debr. 1965, 12, 189-212.

6. Marichal, J.-L. On the associativity functional equation. Fuzzy Sets Syst. 2000, 114, 381-389. [CrossRef]

7. Candeal, J.C.; de Miguel, J.R.; Indurain, E.; Oloriz, E. Associativity equation revisited. Publ. Math. Debr. 1997, 51, 133-144.

8. Ricci, R.C. On the characterization of topological semigroups on closed intervals. Semigr. Forum 2006, 72, 419-432. [CrossRef]

9. Kobayashi, Y.; Nakasuji, Y.; Takahasi, S.-E.; Tsukada, M. Continuous semigroup structures on R, cancellative semigroups and bands. Semigr. Forum 2015, 90, 518-531. [CrossRef]

10. Schweizer, B.; Sklar, A. Probabilistic Metric Spaces; Elsevier North-Holland: New York, NY, USA, 1983.

11. Hadžić, O.; Pap, E. Fixed Point Theory in Probabilistic Metric Spaces; Kluwer Academic Publishers: Dordrecht, The Netherlands, 2001.

12. Alsina, C.; Maurice, F.; Schweizer, B. Associative Functions: Triangular Norms And Copulas; World Scientific: Singapore, 2006.

13. Carruth, J.H.; Hildebrant, J.A.; Koch, R.J. The Theory of Topological Semigroups; Marcel Dekker, Inc.: New York, NY, USA, 1983.

14. Bourbaki, N. Elements of Mathematics, General Topology, Part 2; Addison-Wesley: Don Mills, ON, Canada, 1966.

15. Sierpiński, W. Remarques sur les fonctions de plusieurs variables réelles. Prace Mat. Fiz. 1934, 41, 171-175.

16. Kolmogorov, A.N. On the representation of continuous functions of several variables as superpositions of continuous functions of one variable and addition. Dokl. Akad. Nauk SSSR 1957, 114, 953-956.

17. Rassias, T.M.; Simsa, J. Finite Sums Decompositions in Mathematical Analysis; John Wiley \& Sons Ltd.: Singapore, 1995.

18. Hofmann, K.H. A history of topological and analytical semigroups: A personal view. Semigr. Forum 2000, 61, 1-25. [CrossRef]

19. Arhangel'skii, A.; Tkachenko, M. Topological Groups and Related Structures; Atlantis Press/World Scientific: Paris, France, 2008.

20. Aczél, J.; Dhombres, J. Functional Equations in Several Variables. Volume 31 of Encyclopedia of Mathematics and Its Applications; Cambridge University Press: Cambridge, UK, 1989.

21. Aczél, J. The state of the second part of Hilbert's fifth problem. Bull. Am. Math. Soc. 1989, 20, $153-163$. [CrossRef]

22. Cornfeld, I.P.; Fomin, S.V.; Sinai, Y.G. Ergodic Theory; Springer: Berlin/Heidelberg, Germany; New York, NY, USA, 1982.

23. Biś, A.; Kozłowski, W. On minimal homeomorphisms and non-invertible maps preserving foliations. Topol. Appl. 2019, 254,1-11. [CrossRef]

24. Lin, H.; Phillips, N.C. Crossed products by minimal homeomorphisms. J. Reine Angew. Math. 2010, 641, 95-122. [CrossRef] 
25. Beck, A. Continuous Flows in the Plane; Springer: Berlin/Heidelberg, Germany, 1974.

26. Bajger, M. On the structure of some flows on the unit circle. Aequ. Math. 1998, 55, 106-121. [CrossRef]

27. Zdun, M.C. On embedding of homeomorphisms of the circle in a continuous flow. In Iteration Theory and its Functional Equations, Proceedings of the International Symposium, Schloß Hofen (Lochau), Austria, 28 September-1 October 1984; Lecture Notes Math; Springer: Berlin/Heidelberg, Germany, 1985; Volume 1163, pp. $218-231$.

28. Flouris, K.; Jimenez, M.M.; Debus, J.-D.; Herrmann, H.J. Confining massless Dirac particles in two-dimensional curved space. Phys. Rev. B 2018, 98, 155419. [CrossRef]

29. Brzdęk, J. On continuous solutions of some functional equations. Glas. Mat. 1995, 30, 261-267.

30. Brzdęk, J. On some conditional functional equations of Gołąb-Schinzel type. Ann. Math. Silesianae 1995, 9, 65-80.

31. Brzdęk, J. On the Baxter functional equation. Aequ. Math. 1996, 52, 105-111. [CrossRef]

Publisher's Note: MDPI stays neutral with regard to jurisdictional claims in published maps and institutional affiliations.

(C) 2020 by the authors. Licensee MDPI, Basel, Switzerland. This article is an open access article distributed under the terms and conditions of the Creative Commons Attribution (CC BY) license (http:/ / creativecommons.org/licenses/by/4.0/). 